\title{
Technical Remedies for the Severely Deformed Foot in Leprosy*
}

\author{
LUTZ V. WOLLSTEIN \\ Schieffelin Leprosy Research Sanatorium, \\ Karigiri, Via Katpadi, North Arcot District, S. India
}

\begin{abstract}
this further paper from the orthopaedic workshop the author describes a second walking aid for the leprosy patient with a severely deformed foot, or feet. It can be made from locally available material, costs less than the stay in hospital which would be otherwise necessary, and moreover gives the patient earlier freedom of movement.
\end{abstract}

While it is widely accepted that the priority in treating leprosy patients lies in the field of prevention, it is still the leprosy patient with a severe foot deformity and consequent recurrent ulceration that draws a considerable amount of money away from the funds allotted for the treatment of leprosy. Such work is essential, but largely ineffective if not backed up by a base hospital which can care for acute and chronic cases. It is at the base hospital, then, that the beds are very soon filled up with patients with severe foot problems. In many of these patients the feet are in such a condition that even with intensive teaching, provision of moulded shoes or sandals, and the best co-operation of the patient himself, the problem is still not solved. The five most common foot problems are as follows:

(1) Feet which are grossly absorbed. The weight-bearing area which remains is too small to tolerate normal use of the foot. The stress of (the body weight's) deceleration and acceleration forces is a common cause of re-ulceration in such cases.

(2) Severely deformed feet for which surgery is not indicated, either because of a recent episode of osteomyelitis, fractures or ulceration, or because the patient is unwilling to have the operation, or is medically unfit for correction of the deformity (Fig. 1).

(3) Combined deformity and absorption to such an extent that the "foot-stump" is totally unfit to tolerate the weight of the body in normal walking, even if the stresses of deceleration and acceleration were eliminated (Fig. 2).

(4) A recent history of fracture or of tarsal bone disintegration without concurrent ulceration (Fig. 3).

(5) Destruction of the os calcis (Fig. 4).

* Received for publication 26 April, 1972. 


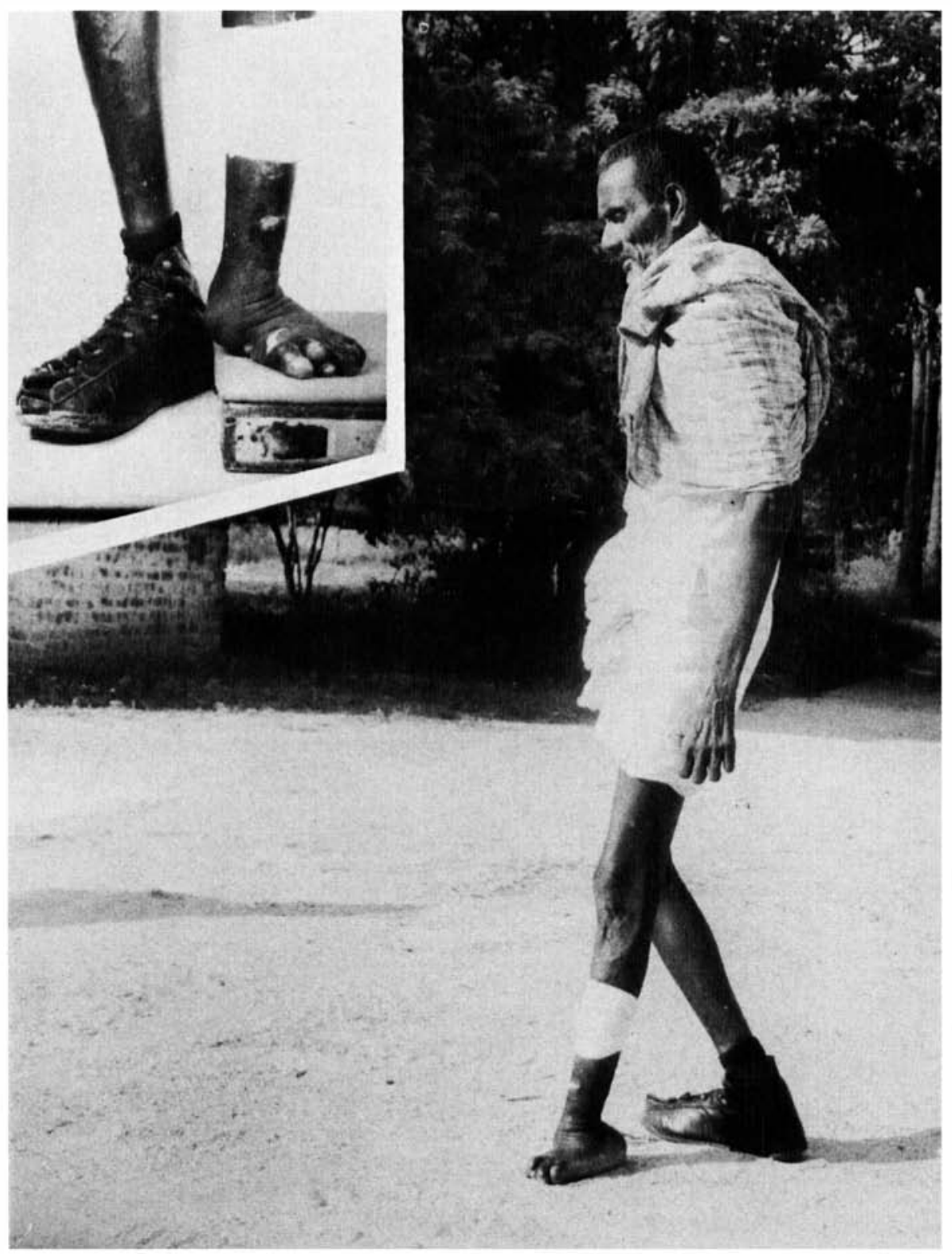

Fig. 1 
L. V. WOLLSTEIN

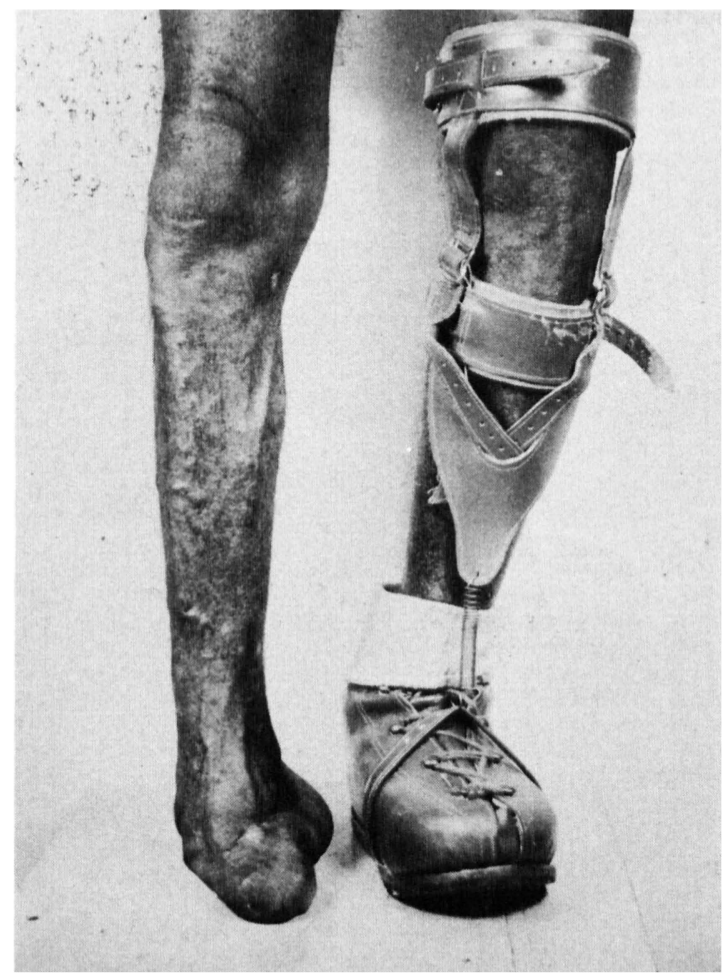

Fig. 2

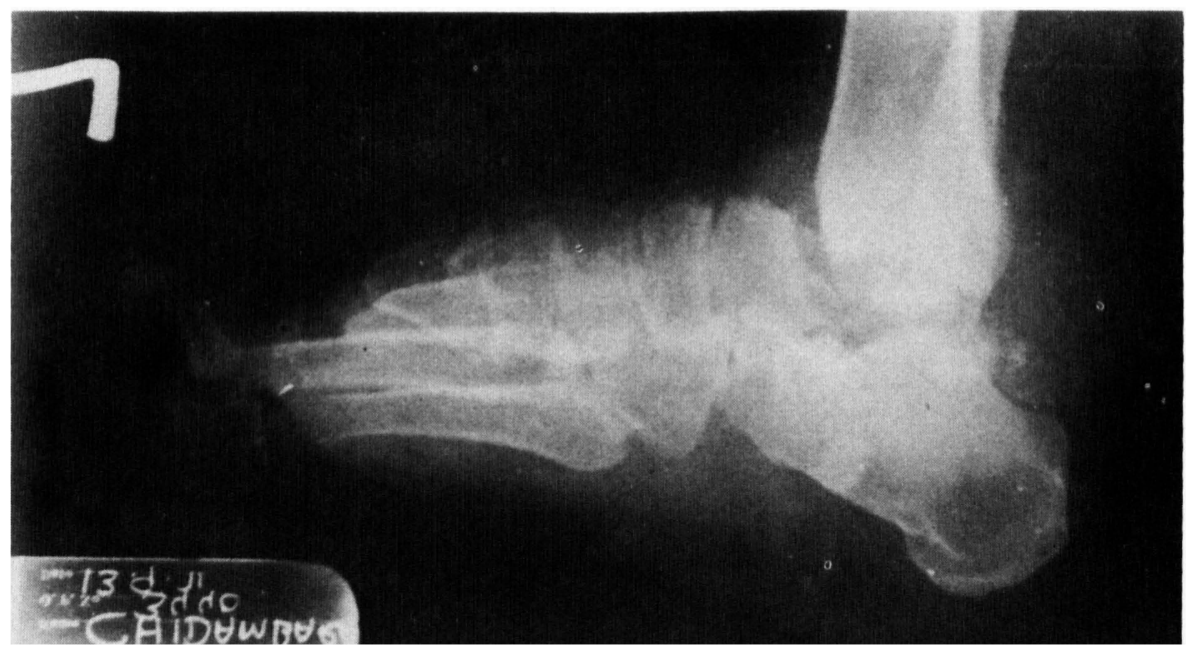

Fig. 3 


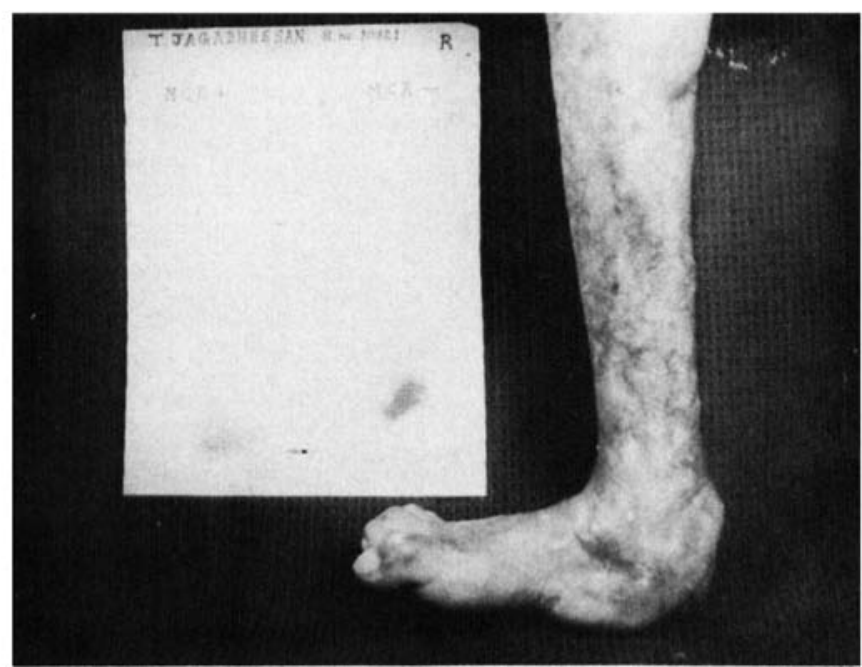

Fig. 4

These deformities are always closely linked with diminished or absent sensation. Unless an above-knee amputation is performed, the problem of weight-bearing by anaesthetic skin remains. It is only transferred to a more proximal area.

If our aim is to make the Leprosy Home a hospital, what are we doing with the group of patients just described above? Any hospital will be fully occupied by inmates like these within a short period. During the last 4 years various experiments have been carried out at the Schieffelin Leprosy Research Sanatorium, Karigiri, in order to find solutions for these foot problems. The aims of our approach have been:

(1) To prevent recurrent ulceration and/or further tarsal disintegration in the feet.

(2) To design orthopaedic appliances which can be manufactured with locally available material.

(3) To provide aids which are inexpensive in comparison with the cost of keeping a patient in hospital for some time because of ulcer problems.

(4) To provide appliances which keep the patient ulcer-free and yet permit him to carry out his daily activities.

(5) To stimulate through this work possible professional rehabilatation for the patients.

(6) To demonstrate that in many cases amputations are not necessary for rehabilitation purposes.

The results of our efforts have been 2 types of caliper, which we have developed towards standardization, viz. the fixed ankle brace walker and the patella-tendon bearing shell.

THE FIXED ANKLE BRACE (FAB) WALKER (Fig. 5)

This type of caliper has been a real break-through in dealing with various kinds of foot problems. Its design is based on the experience gathered over many years 


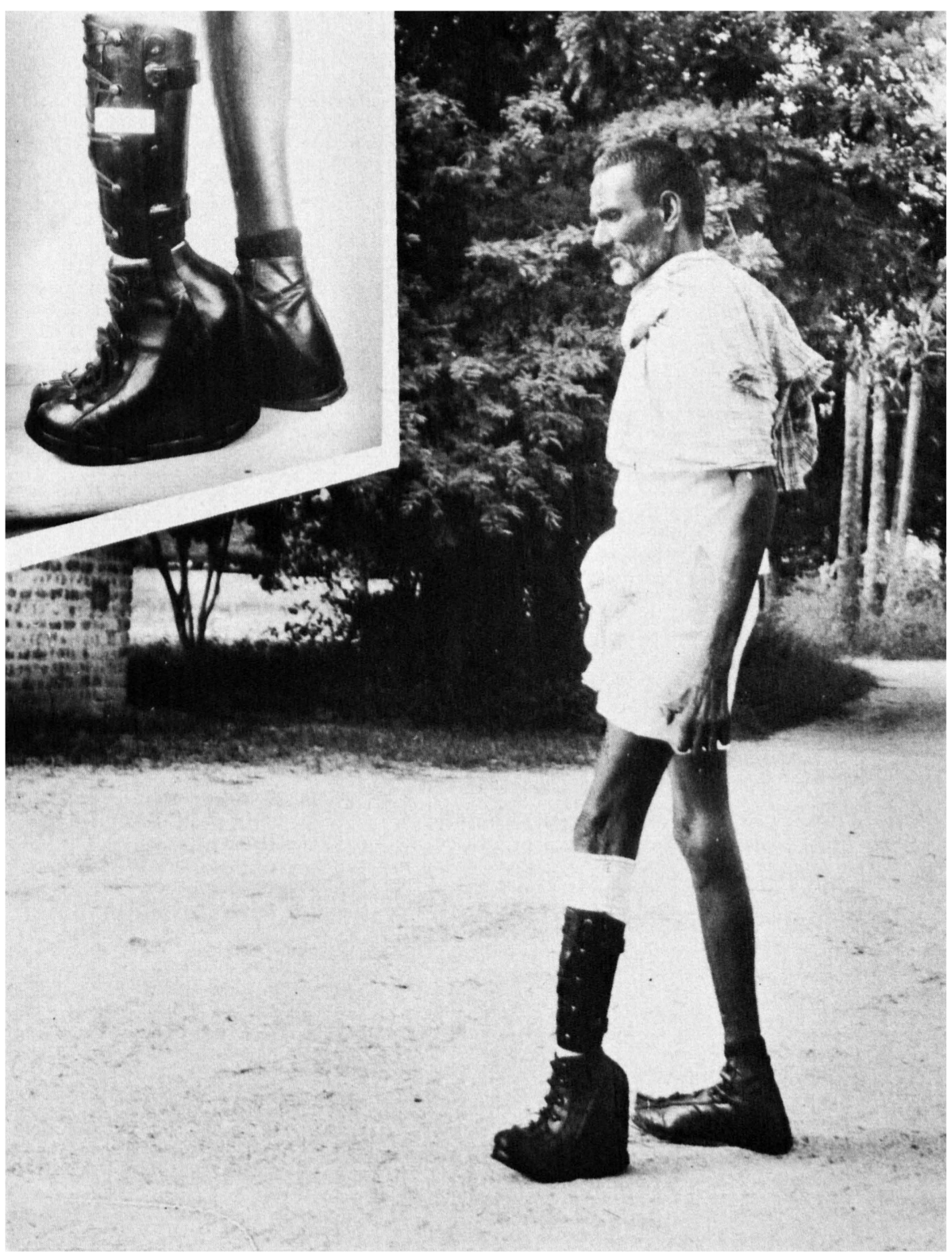

Fig. 5 
with the application of Plaster of Paris (PoP) for ulcer treatment, and was fully described in this journal (Lepr. Rev., 1971, 42, 158).

While the patient with extensively deformed feet remains ulcer-free in a PoP cast which is accurately moulded to the foot, he does not however remain ulcer-free in an equally accurately moulded shoe. The reason for this is that while wearing a moulded shoe, during the walking cycle the foot experiences selected high pressures, especially during deceleration (heél-strike) and acceleration (push-off). These forces are exerted on 2 limited weight-bearing areas of the foot, namely the heel and the metatarsal heads, which in addition may already be scarred or adversely affected by underlying fragmented bones. Tissue trauma and necrosis are common results. Often such a foot may also have lateral instability or be imbalanced (inversion of eversion deformity), for which a moulded boot provides no remedy. Our splint utilizes the same principle as the PoP cast, immobilizing the foot, transferring the walking cycle to a bottom roll, and assuring almost complete contact of the mould to the foot during the entire walking cycle. In addition, the foot is laterally stabilized and well protected from external trauma. In cases of recent failure the significance of this splint is its prolonged protection of the tarsal or metatarsal bones during the acceleration phase. It has been our experience that patients can safely use ordinary footwear after having been protected with a FAB Walker for a period of 12 months. On the other hand it has been quite common for patients with heel or mid-tarsal fractures, af ter discharge from hospital without such an aid, to return later with an even more serious condition.

\section{THE PATELLA TENDON BEARING (PTB) SHELL}

In feet in which tissue and bone absorption is so great that in spite of accurate moulding the plantar surface is still not fit to tolerate the patient's weight, an attempt is made to transfer the weight partially to the condyles of the tibia and patella-tendon area of the leg. The apparatus designed is called the Patella Tendon Bearing (PTB) Shell (Fig. 6).

The specially designed FAB Walker and PTB Shell ensure the possibility of full knee flexion when squatting, since we found that this was one of the chief essentials in persuading the patient to accept this type of footwear. The PTB Shell (Fig. 7) shows an area posteriorly cut-out and deep enough to allow full flexion of the knee. Furthermore, it was essential to construct the PTB Shell in such a way that the dimension for weight-bearing cannot be altered while the patient is taking off or putting on his Shell. The assurance of correct weight distribution must not be governed by the patient's ability or disability in putting on his splint.

\section{POST-FITTING CARE}

While all other patients with normal sensitivity will quickly respond to the discomfort of high pressure caused by an appliance, a leprosy patient with anaesthesia cannot so respond. It is therefore essential that every patient be carefully checked for a period of 6 weeks in order to ensure that no major problems will arise as a result of prolonged wearing of his appliance. To this end we have established the following routine. For 2 weeks the patient is seen twice daily, forenoon and afternoon, at the workshop. The pattern of the stockinette he wears will show as an impression on his skin and indicate whether these patterns are occurring over areas suitable or unsuitable for weight-bearing. Immediate remedies in case of blister formation or skin abrasion are applied. 


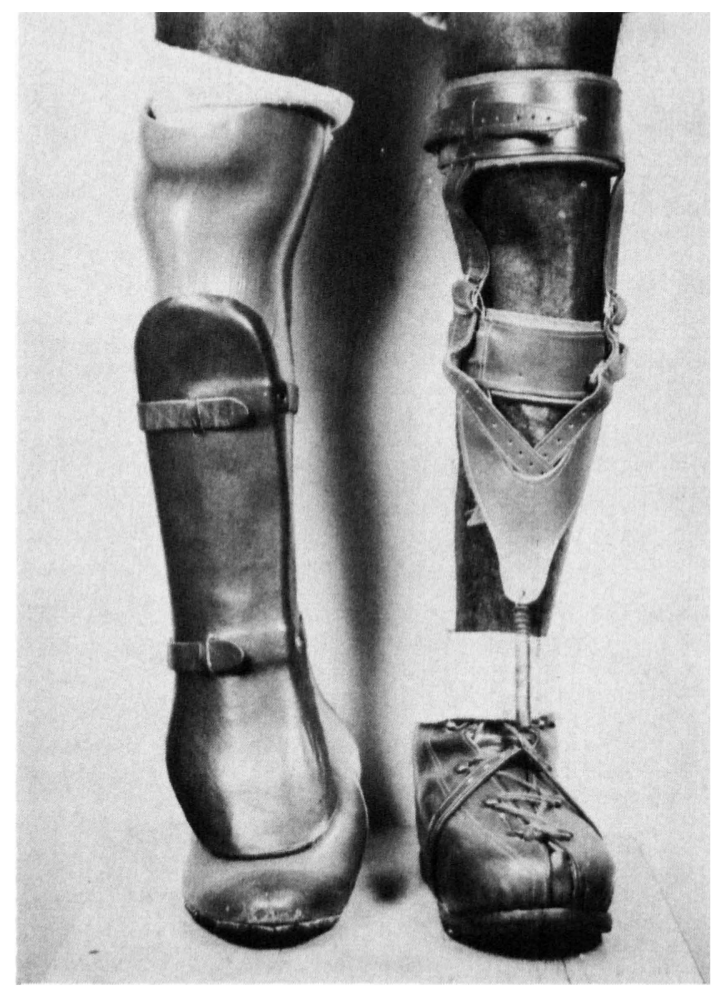

Fig. 6

After a period of 2 weeks, during which the patient is doing only normal everyday activities, he is sent for a so-called "stress-walk". In this, according to his general physical ability, he will have to cover a distance of between 1 and 4 miles daily. This is to test the functioning of the appliance under conditions which the patient is likely to meet in a rural surrounding. It is planned in the form of graduated activity, starting with 2 miles the first day and progressively increasing as long as no problems arise. If any evidence of high pressure develops, the area concerned can be at once relieved of this pressure before ulceration causes irreversible damage.

\section{MATERIALS USED}

In most of our cases we have used locally available materials such as upper-leather, sole-leather, mild steel, and waste-tyre. For the PTB shells stockinette impregnated with polyester resin is used. While the leather material is cheaper it is not very long lasting, whereas resin, though a little more expensive, lasts longer and is relatively free from need of repair.

\section{CONCLUSION}

The average costs for the above described orthopaedic aids range between 10 and 20 dollars (USA), which is approximately equal to the cost of keeping a 


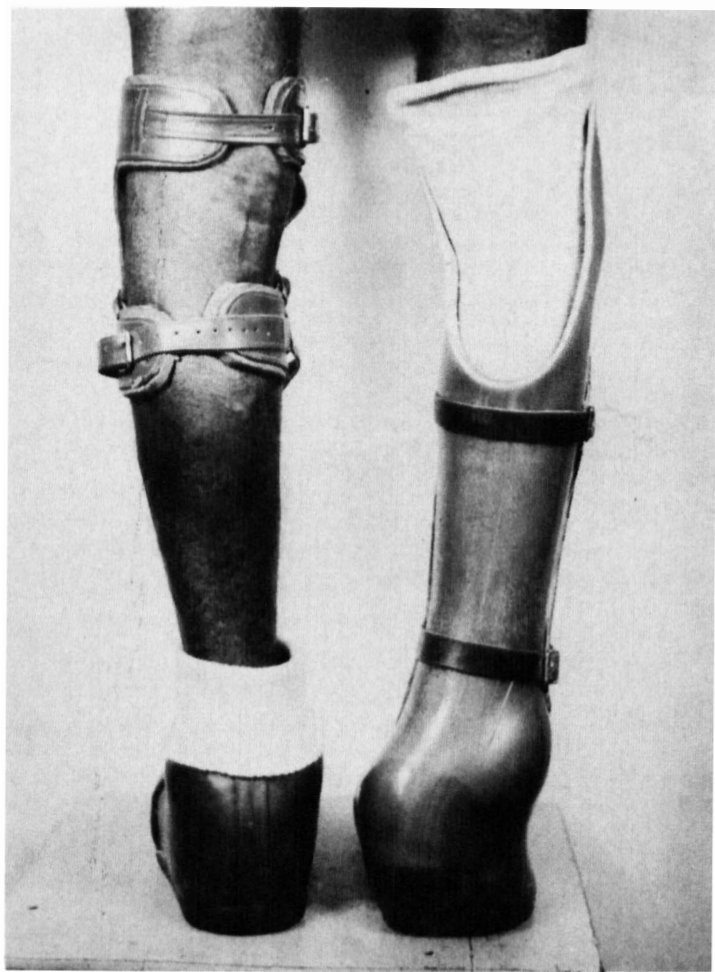

Fig. 7

patient for 18 to 36 days in a hospital in South India. The author hopes to have demonstrated that funds spent for rehabilitation projects are actually helping to save money, i.e., by making it possible to discharge patients who without the service of orthopaedic workshops would otherwise be unfit for discharge. What it means to the individual patient to be able to live his life independent of a hospital cannot of course be expressed in terms of money. 